\title{
Análisis
}

\section{La revolución de los medios informativos en internet. El caso de los contenidos económicos.}

\author{
Por Toni González-Pacanowski
}

\begin{abstract}
Resumen: El concepto de diario electrónico experimenta una metamorfosis permanente originada por la continua transformación del modo de estructurar la información en el entorno digital. Las nuevas audiencias vinculadas al ciberperiodismo demandan igualmente contenidos hipersegmentados con altos niveles de actualidad y basados en estándares de participación colectiva. El ámbito de la información económica es un ejemplo que evidencia ambos procesos, lo que se observa en el mayor peso que adquieren las plataformas de distribución de noticias y contenidos con niveles de popularidad y uso superiores incluso al de los de los mismos medios electrónicos. Este artículo pretende ayudar a entender el cambio que se ha originado desde 2007 y describir el escenario en el que se tendrá que competir asumiendo nuevas coordenadas en el universo mediático de internet.
\end{abstract}

Palabras clave: Periodismo electrónico, Ciberperiodismo, Diario electrónico, Internet, Información económica, Yahoo, Google, Nielsen, ComScore.

Title: The revolution of mass media on the internet. The case of the economic content

Abstract: The electronic newspaper concept undergoes a permanent metamorphosis caused by the continuing transformation of the information structure in the digital environment. The new public audience for cyberjournalism demands updated and hypersegmented content based on collective participation. Economic information is an example that shows both processes, which can be seen with the success and popularity of the platforms for news distribution. This article aims to help understand the change produced since 2007 and tries to describe the scene in which cybermedia will have to compete, assuming new coordinates in the media universe of the internet.

Keywords: Electronic journalism, Cyberjournalism, Electronic newspaper, Internet, Financial information, Economic information, Yahoo, Google, Nielsen, ComScore.

González-Pacanowski, Toni. "La revolución de los medios informativos en internet. El caso de los contenidos económicos”. En: El profesional de la información, 2008, v. 17, n. 5, septiembre-octubre, pp. 533-539.

DOI: 10.3145/epi.2008.sep.07

\section{Escenario}

¿DÓNDE ESTÁ EL DIFERENCIAL que ofrece un medio de comunicación en internet respecto a un buscador como Google o Yahoo? ¿existen diferentes tipos de lectores de información en internet? La gran red ha roto las reglas de dónde, cómo y cuándo debe estar localizada y estructurada la información de actualidad.

No existen límites y las estadísticas demuestran que son los grandes portales y buscadores en internet los que se llevan la mayor parte de la audiencia y sin necesidad de tener marca de medio de comunicación. Nadie es cautivo de una cabecera y cada lector configura su propio microcosmos informativo. Hay un nuevo escenario al que se enfrentan diariamente medios, agencias de publicidad y empresas de medición. En él, los usuarios cambian diariamente de hábitos e hiperseleccionan sus inputs informativos en función de su vida interactiva digital. Una nueva especie de ciberlector centrado en hacer eficiente su información con tecnologías cada vez más sofisticadas y con una identidad más relevante a través de nuevas y más sensibles comunidades digitales.

La fórmula informativa que hoy se considera innovadora en internet, mañana estará obsoleta. La información que se muestra en nuestra pantalla habrá pasado al archivo o eliminada de un servidor en breves instantes. La realidad informativa es un permanente flujo de datos y hechos que se producen bajo el criterio de la máxima inmediatez. Este aluvión de contenido realmente genera reacciones en cadena. El medio se ha convertido en un elemento más que activo, reac- 
tivo para la sociedad y más social que nunca.

No vivimos únicamente el cambio de la naturaleza de un soporte tecnológico, sino la metamorfosis del mismo modo de comunicar. Las reglas del juego han cambiado. La autoridad del profesional de la información requiere contar con el aval de lo que hasta ese momento era una audiencia anónima, y sin apenas poder de reacción ante el bastón de mando de las empresas mediáticas. Es la llegada del metalector-usuario. El receptor utiliza varios canales, de diversa capacidad de actualización y al mismo tiempo administra y colabora en el perfeccionamiento del mensaje. Emisor y receptor adoptan una visión de 360 grados, desde todos los canales posibles con todas las interacciones viables tecnológicamente.

Se precisan periodistas con capacidad para contar historias y adaptarlas a los nuevos formatos digitales. Los testimonios ya no figuran sólo entre comillas, sino incrustados entre documentos audiovisuales y en continua actualización. El periodismo electrónico se fusiona con el digital.

¿Hemos tocado techo con el papel? El mercado informativo de celulosa desde el punto de vista empresarial y cultural todavía tiene un camino que recorrer. En España durante 2006 se vendieron al día 94 ejemplares de periódicos por cada 1.000 habitantes. La cifra está por debajo de los 100 ejemplares que considera la Unesco como umbral del desarrollo. En la Europa de los 15 solamente Portugal registra índices aún más bajos.

Por otro lado, la planificación publicitaria nos dice que la efectividad tiene dos caras. De hecho insertar una campaña publicitaria en periódicos en papel y digitales simultáneamente incrementa su notoriedad entre 15 y 20 puntos porcentuales ${ }^{1}$. La Asociación de Editores de Diarios Españoles (AEDE) es optimista respecto a internet, pero el papel sigue ocupando su puesto de principal soporte. No obstante, algunos editores ya vislumbran que puede existir una naufragio y consideran que va siendo el momento de ponerle el salvavidas a la celulosa.

\section{http://www.aede.es/}

\section{El poder digital en España}

El número de lectores de los diarios digitales alcanzó en 2007 un promedio diario de 5.184.000, un $8,8 \%$ más que el año anterior. El aumento de lectores digitales acumulado desde 2001 se elevaba al 140\%, según datos del Estudio General de Medios. La misma fuente señala que los usuarios únicos de esos periódicos digitales fueron 1.045.093 personas al día, un 51,4\% más que el año anterior. El perfil de los lectores de prensa digital es más joven que el de la prensa en papel, de clase social algo más alta, con un nivel de instrucción sensiblemente más alto y predominantemente varón, con un $64,8 \%$ del total. La búsqueda de información, las noticias y la banca son las principales consultas online desde el trabajo.

\section{http://www.aimc.es/aimc.php}

El informe de Nielsen//NetRatings de 2007 muestra que casi 9 de cada 10 españoles (87\%) que se conecta a internet desde el trabajo accede, como contenido preferente, a las noticias de los principales periódicos online con el fin de informarse de la actualidad en tiempo real. Los internautas realizaron un promedio de más de 17 visitas a este tipo de contenidos durante el mes de marzo de 2008. Según el panel de trabajo de Nielsen, el $82 \%$ de los españoles reconoce la prensa digital como su canal favorito para mantenerse al día de los acontecimientos nacionales e internacionales mientras se encuentra en la oficina, por la rapidez y facilidad que este medio ofrece para conectarse con un solo click. http://www.nielsen-netratings. com/intl.jsp? country=es

\section{Cadena de valor virtual del sector de distribución de noticias}

La etapa de conceptualización o creación es la más valiosa en lo que se ha denominado la cadena de valor virtual de la distribución de noticias $^{2}$. Hoy la infografía multimedia convive con el informador, al mismo tiempo que coordina su edición con la grabación de podcast y vídeo. El lector quiere ver todos los ángulos posibles, o al menos tener la capacidad de seleccionar. No todos los contenidos son susceptibles de tener multiformatos, pero sí es posible en numerosas ocasiones planificar el trabajo de la redacción para lograr un resultado lo más "web" posible. La principal limitación que puede surgir es la coordinación de los diferentes lenguajes multimedia, además de la presupuestaria. En cualquier caso, la gestión del contenido tendrá como resultado una información más cercana al usuario, con más impacto y por tanto más competitiva frente a otras fuentes menos adaptables.

Los medios de comunicación especializados en economía en internet en España abarcan toda la cadena de producción. Generan contenido propio hasta la distribución del mismo entre sus usuarios. Existe una modalidad de portales verticales tipo Invertia o Ecobolsa que ofrecen información-servicio. Su público objetivo es el inversor, y no tanto el convencional de la prensa económica. Analizando el único medio de información económica auditado por OJD interactiva, $w w w$. expansion.com confirma en este sentido que la información bursátil es el contenido rey, muy por encima de otras secciones.

\section{http://www.invertia.com \\ http://www.ecobolsa.com/}

El periodismo económico en internet se enfrenta a retos parecidos a los de otros contenidos periodísti- 
cos presentes en la Red: canibalismo de la edición offline, integración del público participativo/colaborativo, calidad e inmediatez como valores principales de la oferta informativa, distribución en diversidad de formatos y para todas las "pantallas". Hoy ya no se entiende que una agencia no pueda ofrecer sus servicios en los soportes multimedia. Es imprescindible planificar en términos de plataforma multicanal (PC, TV TDT, móvil, PDA, cable) o broadband. Por otro lado entran en escena nuevos distribuidores. Los contenidos económicos para usuarios móviles se especializan y los nuevos distribuidores ya no son propiamente medios de comunicación. Hoy las entidades financieras son plataformas de distribución de bienes y servicios y la información económica se está convirtiendo en una nueva frontera.

Dave Morgan, vicepresidente ejecutivo de estrategia publicitaria de $A O L$, plantea que los modelos de negocio verticales para la edición de noticias no son posiblemente la mejor opción. Tender a una estructura horizontal podría ayudar a clarificar las competencias y definir mejor su visión y misión. En definitiva su nuevo core $^{3}$ :

Los medios se aferran a la difusión del soporte papel. ¿Quiere ello decir que hay "vida" más allá de la celulosa? La World Association of Newspapers (WAN) así lo cree. En su campaña "Get the facts about newspapers" este organismo se posiciona claramente a favor de la defensa del mercado de papel, argumentando que todavía no hay empresa de contenidos en internet que demuestre el vigor y perspectiva de las cabeceras convencionales ${ }^{4}$.

Lo que Google no ha conseguido, y en ello tiene razón la WAN, es impactar del mismo modo que lo hace el papel. Pero ya no se trata de defender un soporte sino de compatibilizarlo con los canales actuales. Un reto que también se une a la necesidad de acertar en el enfoque global de las informaciones, la independencia de los medios económicos, está hoy en entredicho -Rupert Murdoch adquirió el Wall street journal (WSJ) en agosto de 2007, y Louis Vuitton Moet Hennessy (LVMH) el diario Les echos, en diciembre de 2007- y no resulta nada fácil para los responsables de la estrategia publicitaria del medio separar claramente el periodismo de los contenidos enfocados a mantener la reputación corporativa. ¿Pero quién puede negar los más de cincuenta millones de audiencia de los contenidos informativos online? ${ }^{5}$

http://www.larepublica.es/spip. php?article6422

http://www.expansion.com/ edicion/exp/empresas/medios/es/ desarrollo/1072153.html

\section{Ciberdiarios económicos}

El perfil de los ciberdiarios de economía ha cambiado completamente. La arquitectura de la página, la usabilidad y los nuevos lenguajes de programación permiten innovar constantemente. Representan casi el $10 \%$ de los portales de actualidad informativa especializada y suman $49^{6}$. En el sector de los medios económicos según el control de Nielsen, destaca últimamente El economista. En diciembre de 2007 ocupaba la segunda posición, por delante de $E x$ pansión y de Cinco días. No obstante, todos siguen al líder Invertia, que mantiene 735.000 usuarios únicos. Ya.com finanzas y Yahoo finance van a la caza de los diarios tradicionales, lo que supone confirmar la tendencia que se ha vivido en Estados Unidos. Debería clarificarse que los índices de Nielsen no coinciden en el caso de Expansion.com con los de OJD interactiva, que certificaba 612.824 usuarios únicos en diciembre de 2007 (Tabla 1).

Por otro lado, estos indicadores no coinciden con la posición que marca el Page rank (PR) de Google para las cabeceras de los diarios económicos. El resultado ${ }^{7}$ no guarda coincidencia con los datos de Nielsen. Para el gran buscador el orden sería el siguiente: Cinco días (PR 7), Expansión (PR 7), El economista (PR 6), Gaceta de los negocios (PR 6) e Invertia (PR 5).

$\mathrm{Si}$ consideramos la audiencia digital general (Tabla 2, Nielsen, diciembre 2007) en España los líderes son Microsoft y Google con más de 13 millones de visitas cada uno, seguidos de Telefónica (6,4 millones), Yahoo (6,2), France Telecom (5,7), Emule (5,5), Vocento $(5,02)$, Prisacom $(4,3)$ y Wikimedia $(4,3)$. Es evidente que predominan los sitios de servicios -en especial los de correo electrónico- sobre los de noticias. La actividad principal de los usuarios es aprovechar las herramientas de intercomunicación de la Red para satisfacer sus exigencias más sociales.

\section{5. ¡Certifíqueme esas páginas!}

No se ha constituido hasta la fecha un organismo certificador único e indiscutible de la audiencia en in-

\begin{tabular}{|l|c|c|}
\hline \multicolumn{1}{|c|}{ Usuarios únicos } & noviembre 2007 & diciembre 2007 \\
\hline Invertia & 866 & 735 \\
\hline El economista & 578 & 577 \\
\hline CincoDias.com & 637 & 458 \\
\hline Expansion.com & 700 & 436 \\
\hline Ya.com finanzas & 536 & 398 \\
\hline Yahoo! finance & 398 & 307 \\
\hline
\end{tabular}

Tabla 1. Ciberdiarios económicos

Fuente: Nielsen, enero 2008 


\begin{tabular}{|l|c|c|c|}
\hline \multicolumn{1}{|c|}{$\begin{array}{c}\text { Nombre } \\
\text { compañia }\end{array}$} & $\begin{array}{c}\text { Visitantes } \\
\text { únicos } \\
\text { (en miles) }\end{array}$ & $\begin{array}{c}\text { Reach } \\
\%\end{array}$ & $\begin{array}{c}\text { Tiempo por } \\
\text { persona }\end{array}$ \\
\hline Microsoft & 13.353 & 88,15 & $05: 07: 07$ \\
\hline Google & 13.230 & 87,33 & $01: 19: 48$ \\
\hline Telefónica / Terra & 6.415 & 42,35 & $00: 20: 45$ \\
\hline Yahoo! & 6.211 & 41,00 & $00: 45: 20$ \\
\hline France Telecom & 5.778 & 38,14 & $00: 18: 27$ \\
\hline Emule-Project.net & 5.597 & 36,95 & $01: 17: 33$ \\
\hline Vocento & 5.021 & 33,14 & $00: 13: 43$ \\
\hline RCS MediaGroup & 4.823 & 31,84 & $00: 23: 35$ \\
\hline Prisacom & 4.397 & 29,03 & $00: 16: 58$ \\
\hline Wikimedia Foundation & 4.384 & 28,94 & $00: 14: 04$ \\
\hline
\end{tabular}

Tabla 2. Top 10 sitios web de España

Fuente: Nielsen, diciembre 2007

ternet. Sólo, y no es poco, indicadores orientativos de los buscadores y estudios de consultoras y sus posibles limitaciones. El caso de Alexa (figura 1), si bien sólo representa a un porcentaje de usuarios de internet que se han instalado su aplicación específica de navegación (barra en el navegador), es indicativo para establecer comparaciones. Resulta curioso el contraste que existe entre el líder de la prensa económica, Expansión y El economista. Si nos atenemos a las estadísticas de Nielsen (Tabla 1), el resultado se confirma.

Es evidente que las audiencias en internet todavía tienen que ser sometidas a un proceso de estanda-

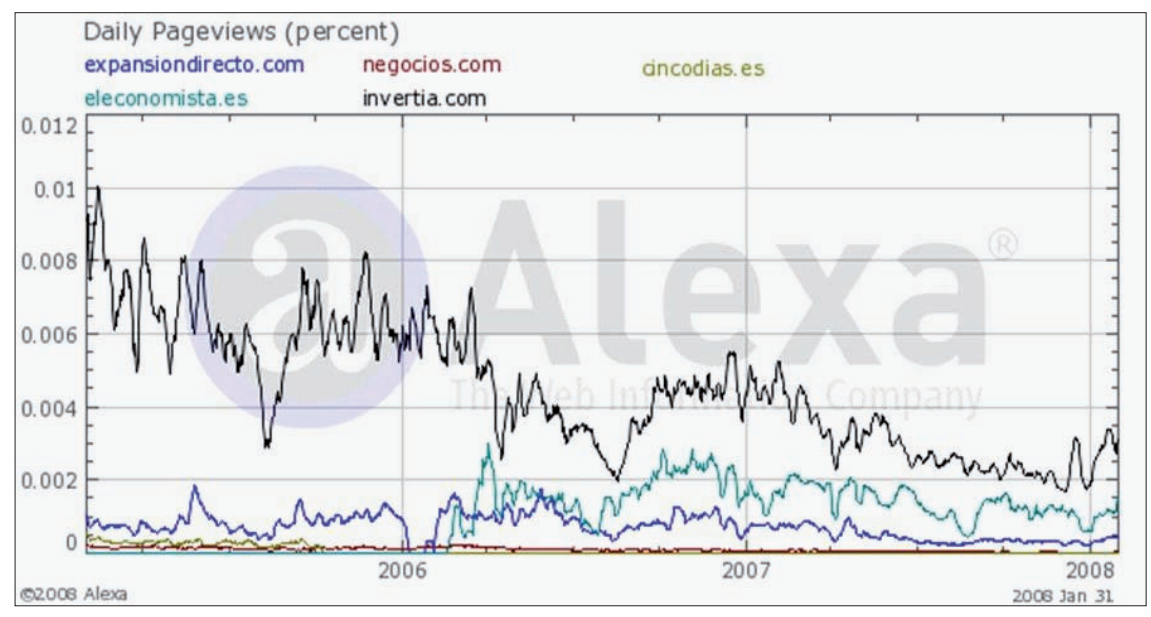

Figura 1. Fuente: Alexa 2008 suele contabilizar como tráfico habitualmente para el medio de origen -puesto que se muestra bajo otra cabecera-, y sólo puede adjudicarse la audiencia si un internauta hace clic en cualquier titular y lo visita. Esta forma de analizar el tráfico puede ser útil para obtener algunas conclusiones, pero no resulta el mejor indicador para describir la audiencia de un medio en internet. Nielsen tuvo que revisar este concepto ante las críticas de los ciberdiarios.

\section{EI intermediario decide}

¿Es posible que los distribuidores lleguen a tener más páginas vistas que los mismos medios? La información de bolsa (figura 2), como sucede con Invertia, el portal de Terra, se ha convertido en un subgénero y con sus propios canales de información al instante. Pese a que no puede competir en noticias, manejan informaciones más interesantes para un inversor, pues las seleccionan con un planteamiento estratégico y no tan informativo.

Los distribuidores o portales verticales se consolidan en Estados Unidos $^{8}$ (figura 3) como una fuente habitual de consulta de noticias económicas. Desde que irrumpieran los medios de comunicación en internet, han cambiado aspectos relativos a la interactividad, velocidad de acceso, servicios específicos de consumo, entre otros aspectos.

En este sentido, según datos de Comscore, en el pasado mes de mayo de 2008 el portal de noticias financieras de Yahoo en Estado Unidos registraba una audiencia anual de 18 millones de visitantes, lo que ha representado un incremento del $58 \%$ respecto al año anterior. Incluso, los otros grandes distribuidores de noticias económicas parecen vivir con la crisis subprime sus mejores momentos con incrementos de audiencia del $48 \%$, en el caso de AOL Money \& Finance o del 13\% como ha sucedido con MSN Money. El público con crecimientos más 


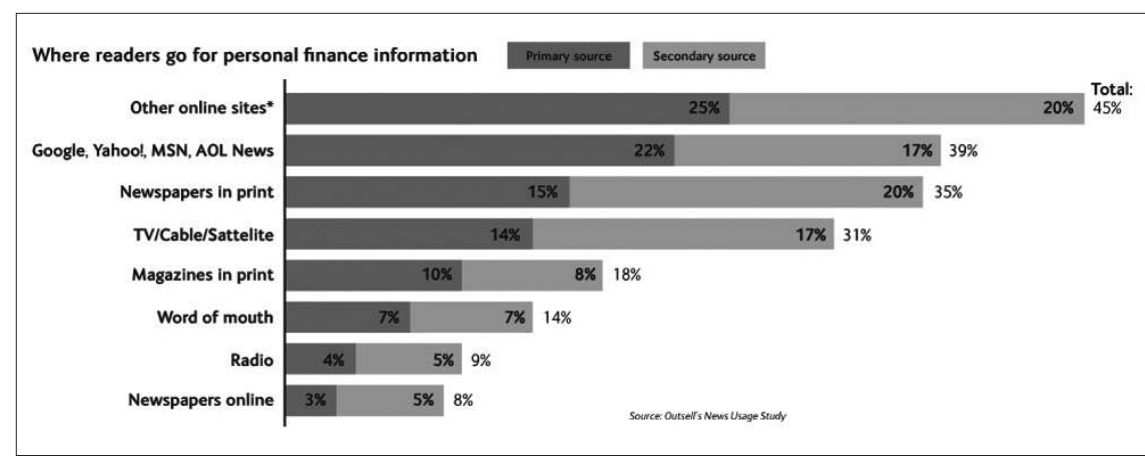

Figura 2. Fuente: Doctor, Ken. Understand what's happening in the media business in $\{11\}$ steps. Society of News Design (SND)

destacables, si se analiza la audiencia de estos "superportales", ha sido el de cincuenta años de edad. Por otro lado, el crecimiento del segmento de audiencia por debajo de la cincuentena ha sido algo inferior.

http://www.comscore.com/press/ release. asp? press $=2317$

Sin embargo, parece que los portales de los diarios económicos tradicionales no están muy dispuestos a luchar por la batalla de generar comunidades masivas de usuarios lectores. Los portales verticales por su lado se han adelantado y no hay quien pueda levantar el tráfico de estas webs ancladas en la filosofía del medio unidireccional si tomásemos como referencia las estadísticas de Alexa. Su principal valor es la integridad de la noticia, pero posiblemente el modelo de distribución y la apuesta por la web social han sido lagunas que no se han planificado correctamente en sus estrategias. Salvo el valor de archivo documental, los diarios económicos en cuanto a noticias no son más competitivos que otros canales intermediarios, en ocasiones más precisos y contundentes en la solidez de sus datos.

Según Ken Doctor es preciso reinventar las posibilidades de los contenidos centrando la atención en lo que hacen los usuarios de la web. El triángulo está conformado por tres ejes. El e-mail, que representa el $53 \%$ de la actividad, las búsquedas que suponen el $38 \%$ y la consulta de noticias que se acerca al $30 \%$.

Con foros y blogs no se puede dar por satisfecho un cibermedio. Debe convertirse en protagonista de la actualidad junto con sus lectores. Hacer las ruedas de prensa interactivas con su audiencia y abrir el despacho de los directores generales a sus propios usuarios. Es el medio el que tiene la posibilidad de trasladar al escenario de los hechos a su lector y hacerle vibrar con la pasión que el mismo periodista siente por realizar su trabajo. Todo ello sin dejar que la sombra de la manipulación o información interesada en aniquilar la voz de los consumidores le comprometa. El redactor jefe de un diario económico tiene que lidiar con colectivos nada dispuestos a ser convencidos por sus redactores con las supuestas excelencias de algunos lanzamientos o beneficios económicos maquillados. ¿Que sucedería si hoy el ordenador de consumo más avanzado del planeta, el Mac Pro de Apple con ocho procesadores y que puede llegar a alcanzar un precio de 25.000 euros tiene problemas según algunos blogs de usuarios; o el coche del año del 2007, el Ford S Max, cumbre de la tecnología del motor, no pareciera muy fiable según foros de internet?

Y así, bajo la información oficial ha nacido una audiencia alternativa que puede dar al traste con la credibilidad de sus mejores expertos en sectores económicos. La audiencia desenmascara la teatralidad y la realidad impuesta simplemente con la huida a otro soporte, a otro medio más atractivo y que le involucre con su opinión en los acontecimientos. El periodismo se ha de convertir en una herramienta verdaderamente participativa para el ciudadano que se moviliza por intereses muy distintos a los de una empresa informativa.

Es posible un nuevo periodismo económico: para ello hay que invertir en la sociabilidad del canal, al mismo tiempo que en tener las mejores plumas de las mejores escuelas de negocios para analizar la realidad económica, y todo ello implica inversión, sobre todo en creatividad, extendida u olvidada detrás de las mesas de la redacción por la organización excesivamente jerárquica de las redacciones, basada en la comunicación interna transversal y desincentivadora de los talentos más jóvenes. Nunca como ahora han salido jóvenes valores preparados y además pertenecientes a la generación web. ¿Por qué no aprovecharlos y reaccionar antes de que un portal de servicios acabe con nuestro modelo de negocio?

\section{Tendencias de la publicidad}

Sabemos que la inversión publicitaria anual en internet ha pasado de 71,2 millones de euros en 2002 a 491,6 millones de euros estimados en 2007, con un incremento anual en torno al $58 \%$, según la previsión de Carat a partir de los datos del Interactive Advertising Bureau (IAB) y de PricewaterhouseCoopers $(P W C)$. Otros indicadores como el procedente del análisis del Grupo Consultores ${ }^{9}$ refleja como la perspectiva es positiva para la inversión en el soporte digital. El estudio se realizó sobre una población de anunciantes de los 400 primeros según inversión en medios en 2006 (InfoAdex) y con todas las agencias de medios. 
http://www.iabspain.net/know. php? $d=k n o$

\section{http://www.infoadex.es}

Su conclusión es clara: internet ocupa el centro de la preocupación y de la estimación de inversión tanto por anunciantes como por agencias de medios. Consolidado como medio, los entrevistados lo perciben muy al alza, como soporte innovador y con nuevos formatos creativos, con alta capacidad y alcance sobre públicos jóvenes. No obstante, es recomendable utilizar fórmulas no intrusivas para el lector, como concluye por su lado $\mathrm{Ca}$ rat Expert (Informe AEDE 2008) ${ }^{1}$.

\section{La generación network}

El colectivo humano forma una network (una red) con capacidad de plantear alternativas en todos los ámbitos; al mismo tiempo puede convertirse en protagonista, salir de un anonimato o mantenerlo sin renunciar a la difusión masiva de su conocimiento.

La audiencia que para determinados medios resultaba pasiva y manipulable ante el impacto del medio, se ha autoproclamado periodista de la noche al día. No hay consumidores sino comunidades que se comportan como un público flotante que puede ir en cualquier dirección y con el canal que más sintonice con sus prioridades del momento. Con la web 2.0 entran en juego lenguajes avanzados de programación que han propiciado la creación de herramientas online más ágiles y sencillas de utilizar y con una enorme capacidad de interrelacionar a sus usuarios, además de estar autogestionadas con la ayuda de aplicaciones inteligentes. El contenido va en busca del lector, más selectivo y al mismo tiempo proactivo, dispuesto a compartir.

Por diferenciación con el estado previo, en la web 2.0 la generación network es creadora y generadora de contenidos, utiliza la web como

\begin{tabular}{|l|l|}
\hline \multicolumn{1}{|c|}{ Web $\mathbf{1 . 0}=$ poseer } & \multicolumn{1}{c|}{ Web $\mathbf{2 . 0}=$ compartir } \\
\hline leer & escribir \\
\hline empresas & comunidades \\
\hline html & xml, ajax, flash \\
\hline páginas personales & blogs, wikis \\
\hline portales & rss \\
\hline formularios & aplicaciones inteligentes \\
\hline módem & adsl, banda ancha, móviles \\
\hline audiencia activa & audiencia proactiva \\
\hline
\end{tabular}

Tabla 3. La generación network

herramienta y al mismo tiempo plataforma de intercambio de información. Vivimos una fase en la que hemos comenzado a interaccionar con la TV, lo que será más realidad con la TDT (TV digital terrestre) y el estándar IPTV (internet protocol $T V$ ) en pocos años. Los dispositivos móviles también están en un punto de asentamiento tecnológico, en donde la interfaz telefónica e informática evoluciona cada vez más hacia una convergencia de funciones.

Pero, ¿alguien da más? Un mayor número de usuarios o volumen de tráfico permite obtener una posicionamiento estratégico y proyectar el modelo de negocio en broadband. La web 2.0 representaba el $2 \%$ del tráfico de internet en el 2005, y alcanzó el $12 \%$ el pasado año. Los movimientos del sector son espectaculares y las ofertas de adquisición parece que han hecho olvidar las penas que se vivieron en la crisis tecnológica después de que AOL comprara Time Warner en el año 2000.

Algunas realidades ineludibles que se presentan a la vuelta de la esquina con la web 2.0 serían las siguientes (Informe Optaros) ${ }^{10}$ :

- Convergencia de los medios: medios diferentes colaboran para crear comunidades de usuarios.

- Sociedad en red: el objetivo es crear social graph (espacios comunes de identidad en la Red).
- Online y offline se confunden: todo tiene estrategia digital.

- La publicidad se reinventa: product placement interactivo.

Para 2010 la cuota de publicidad de los medios online alcanzará el 10,2\%, según datos de PricewaterhouseCoopers $(P W C)$, y superará a la radio y a las revistas. Las estrategias se enfocan claramente al medio online.

Los widgets ${ }^{11}$ se hacen con los espacios de pantalla que quedan libres en la distribución de información y el efecto 2.0 llegará a los espacios profesionales donde el intercambio de información se nutre de sistemas informáticos en ocasiones cerrados a los beneficios de la red internet.

Es evidente que el entorno de evolución es positivo. Si nos atenemos a Jupiter Research, en su informe Worldwide online population forecast, 2007 to 2012. Driving growth through emerging economies, los datos macroeconómicos nos confirman que las nuevas tecnologías viven un momento de crecimiento y hacen pensar que estamos al principio de un ciclo inversor que se puede prolongar por un plazo de años y en el que el momento de crisis no lo marcará precisamente la frescura y energía de las nuevas tecnologías. El caso de China en este sentido, con incrementos espectaculares, abre una vía de 
desarrollo apasionante a la vez que incierta. Los compradores online no paran de aumentar y la inversión y gasto en todo lo que tienda a ser web es prácticamente impredecible en su crecimiento.

El número de usuarios online en todo el mundo aumentará $44 \%$ entre 2007 y 2012, alcanzando 1,8 millones de usuarios. En 2012, una cuarta parte de la población mundial tendrá acceso a internet de manera regular. El cambio será espectacular: Brasil, Rusia, India y China experimentarán algunas de las más altas tasas de crecimiento. En 2011, China superará a los EUA en número de usuarios para convertirse en la principal población online, como indica Jupiter Research.

El crecimiento natural de internet con más usuarios y mayor número de contenidos relativos a la economía requiere al mismo tiempo que se desarrollen progresivamente las capacidades de infraestructura, como la extensión geográfica del ancho de banda suficiente y sin incurrir en el aumento de la brecha digital entre sociedades y culturas.

Los lenguajes html y xhtml se mantendrán al menos hasta que todos los sitios web sean capaces de regenerarse y reconvertirse con programaciones acordes con el entorno semántico y la accesibilidad se estandarice para éstos (xml, rdf, owl, spl, etc.).

Sin duda, mientras asistimos a la revolución de los contenidos autoproducidos y sociales en la Red que conforman cada día una comunidad nueva, la web se despojará de su dinámica mecánica basada en la expresión morfológica y sintáctica del lenguaje humano, para adaptarse a la expresión semántica, más inteligente y eficaz en sus resultados dentro de los buscadores y en la gestión global de la información.

Establecer un escenario aproximado de lo que puede ser la web

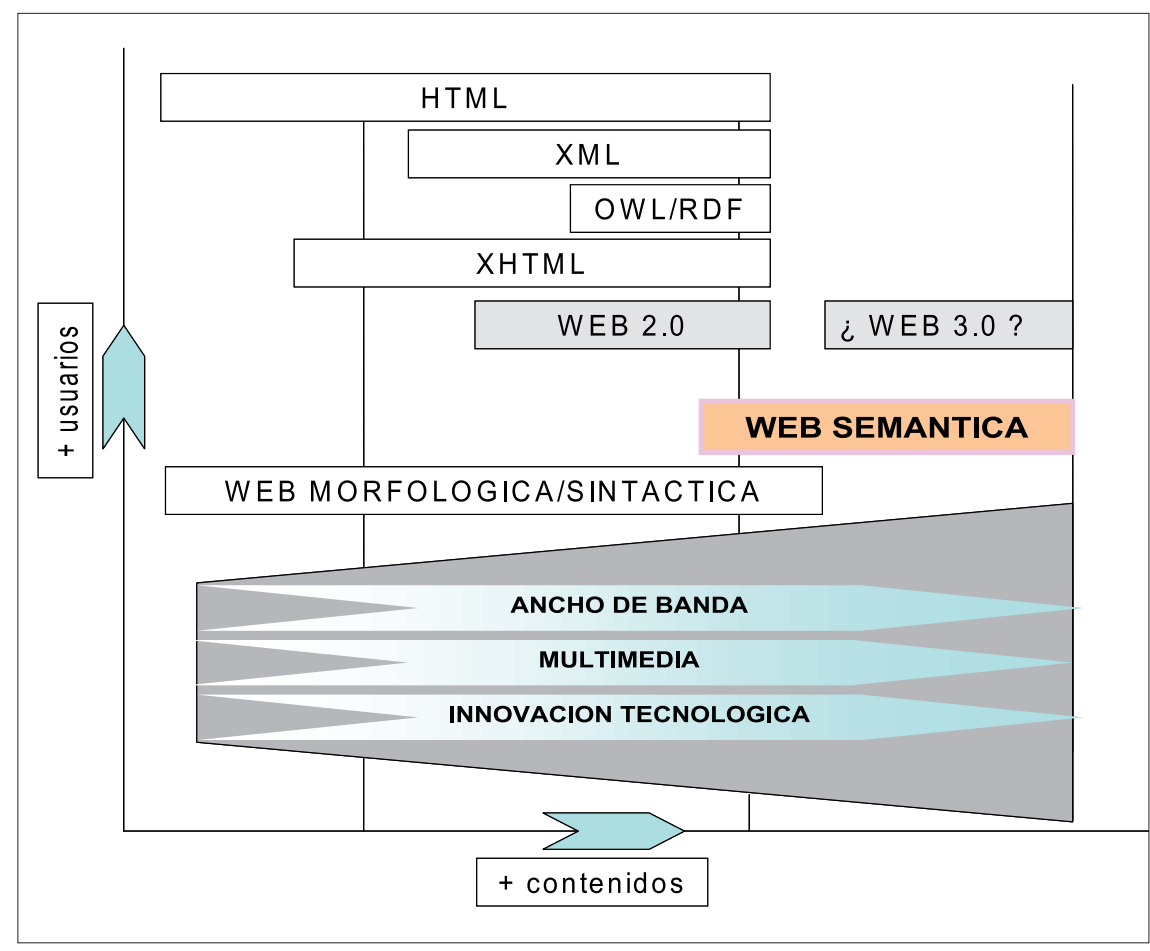

Figura 4

semántica en el ámbito de la información económica en los próximos diez años es aventurado, pues se trata no sólo de un cambio y asentamiento tecnológico, sino de un giro mucho más global y abierto, donde se requiere la implicación de los principales agentes que validan y consensuan el conocimiento relacionado con la economía (figura 4).

\section{Notas}

1. Carat Expert e Instituto de investigación $\mathrm{Ci}$ mec Millward Brown. En: Libro blanco de la prensa diaria 2008, AEDE 2008.

http://www.aede.es/documentos/notaprensa 2008.doc

2. Análisis de la creación de valor en el ebusiness. Una aplicación al sector de distribución de noticias online. Boletín económico de ICE, 2006, n. 2885, p. 53.

3. Morgan, Dave. Consultado el 29-01-2008. "Most newspapers don't have a core competency. They have several. They are much closer to vertical monopolies than they are to point solution providers. They are not just news gatherers and reporters and editors. They are printing companies. They are distribution companies. They are ad sales companies. They are direct marketing companies. They are digital media companies. Many are good at all of these functions. Digital competition and audience fragmentation are fracturing the business models that have built these great, vertically integrated companies. While they are clearly not sustainable as they are, they might be quite sustainable in horizontal pieces". http://blogs.mediapost.com/spin/?p=1197
4. "Adivinanza ¿quién llega a 1.600 millones de personas por día?". WAN newsletter, n. 37, febrero 2008 , p. 9.

http://www.wan-press.org/article 17659.html http://www.wan-press.org/article17116.html

5. "Good news, bad news for papers". Wired. Consultado el 15-01-2008.

http://www.wired.com/culture/lifestyle/news/ 2006/10/71904

6. Salaverría, Ramón (coord.). Cibermedios: el impacto de internet en los medios de comunicación en España. Sevilla: Comunicación Social Ediciones y Publicaciones, 2005.

7. Búsqueda realizada en Google el 1 de marzo de 2008.

8. Doctor, Ken. "Understand what's happening in the media business in $\{11\}$ steps". Society of News Design (SND).

http://www.slideshare.net/jonathonberlin/stateof-the-newspaper-industry/

9. Estudio de calidad de los medios de comunicación en España. $7^{\mathrm{a}}$ edición, 2007. Grupo Consultores.

10. Informe Optaros 2008.

http://www.marketingdirecto.com/noticias/abre. php? idnoticia $=26284$

11. Un widget es una pequeña aplicación o programa, usualmente presentado en archivos o ficheros pequeños que son ejecutados por un motor de widgets o widget engine. Permiten dar fácil acceso a funciones frecuentemente usadas y proveer de información visual.

Toni González-Pacanowski, Tecnología de la información y producción periodística, Universitat Abat Oliba, Barcelona.

tonigonza@telefonica.net 